\title{
Bipartite Graph Partitioning and Data Clustering*
}

\author{
Hongyuan Zha \\ Xiaofeng $\mathrm{He}$ \\ Dept. of Comp. Sci. \& Eng. \\ Penn State Univ. \\ State College, PA 16802 \\ \{zha,xhe\}@cse.psu.edu
}

\author{
Chris Ding \\ Horst Simon \\ NERSC Division \\ Berkeley National Lab. \\ Berkeley, CA 94720 \\ \{chqding,hdsimon\}@lbl.gov
}

\author{
Ming Gu \\ Dept. of Math. \\ U.C. Berkeley \\ Berkeley, CA 94720 \\ mgu@math.berkeley.edu
}

\begin{abstract}
Many data types arising from data mining applications can be modeled as bipartite graphs, examples include terms and documents in a text corpus, customers and purchasing items in market basket analysis and reviewers and movies in a movie recommender system. In this paper, we propose a new data clustering method based on partitioning the underlying bipartite graph. The partition is constructed by minimizing a normalized sum of edge weights between unmatched pairs of vertices of the bipartite graph. We show that an approximate solution to the minimization problem can be obtained by computing a partial singular value decomposition (SVD) of the associated edge weight matrix of the bipartite graph. We point out the connection of our clustering algorithm to correspondence analysis used in multivariate analysis. We also briefly discuss the issue of assigning data objects to multiple clusters. In the experimental results, we apply our clustering algorithm to the problem of document clustering to illustrate its effectiveness and efficiency.
\end{abstract}

\section{Categories and Subject Descriptors}

H.3.3 [Information Search and Retrieval]: Clustering; G.1.3 [Numerical Linear Algebra]: Singular value decomposition; G.2.2 [Graph Theory]: Graph algorithms

\section{General Terms}

Algorithms, theory

\section{Keywords}

document clustering, bipartite graph, graph partitioning, spectral relaxation, singular value decomposition, correspondence analysis

\footnotetext{
*Part of this work was done while Xiaofeng He was a graduate research assistant at NERSC, Berkeley National Lab.
}

Permission to make digital or hard copies of all or part of this work for personal or classroom use is granted without fee provided that copies are not made or distributed for profit or commercial advantage and that copies bear this notice and the full citation on the first page. To copy otherwise, to republish, to post on servers or to redistribute to lists, requires prior specific permission and/or a fee.

CIKM '01 November 5-10, 2001, Atlanta, Georgia. USA

Copyright 2001 ACM X-XXXXX-XX-X/XX/XX ...\$5.00.

\section{INTRODUCTION}

Cluster analysis is an important tool for exploratory data mining applications arising from many diverse disciplines. Informally, cluster analysis seeks to partition a given data set into compact clusters so that data objects within a cluster are more similar than those in distinct clusters. The literature on cluster analysis is enormous including contributions from many research communities. (see $[6,9]$ for recent surveys of some classical approaches.) Many traditional clustering algorithms are based on the assumption that the given dataset consists of covariate information (or attributes) for each individual data object, and cluster analysis can be cast as a problem of grouping a set of $n$-dimensional vectors each representing a data object in the dataset. A familiar example is document clustering using the vector space model [1]. Here each document is represented by an $n$-dimensional vector, and each coordinate of the vector corresponds to a term in a vocabulary of size $n$. This formulation leads to the so-called term-document matrix $A=\left(a_{i j}\right)$ for the representation of the collection of documents, where $a_{i j}$ is the so-called term frequency, i.e., the number of times term $i$ occurs in document $j$. In this vector space model terms and documents are treated asymmetrically with terms considered as the covariates or attributes of documents. It is also possible to treat both terms and documents as first-class citizens in a symmetric fashion, and consider $a_{i j}$ as the frequency of co-occurrence of term $i$ and document $j$ as is done, for example, in probabilistic latent semantic indexing [12]. ${ }^{1}$ In this paper, we follow this basic principle and propose a new approach to model terms and documents as vertices in a bipartite graph with edges of the graph indicating the co-occurrence of terms and documents. In addition we can optionally use edge weights to indicate the frequency of this co-occurrence. Cluster analysis for document collections in this context is based on a very intuitive notion: documents are grouped by topics, on one hand documents in a topic tend to more heavily use the same subset of terms which form a term cluster, and on the other hand a topic usually is characterized by a subset of terms and those documents heavily using those terms tend to be about that particular topic. It is this interplay of terms and documents which gives rise to what we call bi-clustering by which terms and documents are simultaneously grouped into semantically co-

\footnotetext{
${ }^{1}$ Our clustering algorithm computes an approximate global optimal solution while probabilistic latent semantic indexing relies on the EM algorithm and therefore might be prone to local minima even with the help of some annealing process.
} 
herent clusters.

Within our bipartite graph model, the clustering problem can be solved by constructing vertex graph partitions. Many criteria have been proposed for measuring the quality of graph partitions of undirected graphs [4, 14]. In this paper, we show how to adapt those criteria for bipartite graph partitioning and therefore solve the bi-clustering problem. A great variety of objective functions have been proposed for cluster analysis without efficient algorithms for finding the (approximate) optimal solutions. We will show that our bipartite graph formulation naturally leads to partial SVD problems for the underlying edge weight matrix which admit efficient global optimal solutions. The rest of the paper is organized as follows: in section 2 , we propose a new criterion for bipartite graph partitioning which tends to produce balanced clusters. In section 3, we show that our criterion leads to an optimization problem that can be approximately solved by computing a partial SVD of the weight matrix of the bipartite graph. In section 4, we make connection of our approximate solution to correspondence analysis used in multivariate data analysis. In section 5 , we briefly discuss how to deal with clusters with overlaps. In section 6 , we describe experimental results on bi-clustering a dataset of newsgroup articles. We conclude the paper in section 7 and give pointers to future research.

\section{BIPARTITE GRAPH PARTITIONING}

We denote a graph by $G(V, E)$, where $V$ is the vertex set and $E$ is the edge set of the graph. A graph $G(V, E)$ is bipartite with two vertex classes $X$ and $Y$ if $V=X \cup Y$ with $X \cap Y=\emptyset$ and each edge in $E$ has one endpoint in $X$ and one endpoint in $Y$. We consider weighted bipartite graph $G(X, Y, W)$ with $W=\left(w_{i j}\right)$ where $w_{i j}>0$ denotes the weight of the edge between vertex $i$ and $j$. We let $w_{i j}=0$ if there is no edge between vertices $i$ and $j$. In the context of document clustering, $X$ represents the set of terms and $Y$ represents the set of documents, and $w_{i j}$ can be used to denote the number of times term $i$ occurs in document $j$. A vertex partition of $G(X, Y, W)$ denoted by $\Pi(A, B)$ is defined by a partition of the vertex sets $X$ and $Y$, respectively: $X=$ $A \cup A^{c}$, and $Y=B \cup B^{c}$, where for a set $S, S^{c}$ denotes its compliment. By convention, we pair $A$ with $B$, and $A^{c}$ with $B^{c}$. We say that a pair of vertices $x \in X$ and $y \in Y$ is matched with respect to a partition $\Pi(A, B)$ if there is an edge between $x$ and $y$, and either $x \in A$ and $y \in B$ or $x \in A^{c}$ and $y \in B^{c}$. For any two subsets of vertices $S \subset X$ and $T \subset Y$, define

$$
W(S, T)=\sum_{i \in S, j \in T} w_{i j}
$$

i.e., $W(S, T)$ is the sum of the weights of edges with one endpoint in $S$ and one endpoint in $T$. The quantity $W(S, T)$ can be considered as measuring the association between the vertex sets $S$ and $T$. In the context of cluster analysis edge weight measures the similarity between data objects. To partition data objects into clusters, we seek a partition of $G(X, Y, W)$ such that the association (similarity) between unmatched vertices is as small as possible. One possibility is to consider for a partition $\Pi(A, B)$ the following quantity

$$
\begin{aligned}
\operatorname{cut}(A, B) & \equiv W\left(A, B^{c}\right)+W\left(A^{c}, B\right) \\
& =\sum_{i \in A, j \in B^{c}} w_{i j}+\sum_{i \in A^{c}, j \in B} w_{i j} .
\end{aligned}
$$

Intuitively, choosing $\Pi(A, B)$ to minimize cut $(A, B)$ will give rise to a partition that minimizes the sum of all the edge weights between unmatched vertices. In the context of document clustering, we try to find two document clusters $B$ and $B^{c}$ which have few terms in common, and the documents in $B$ mostly use terms in $A$ and those in $B^{c}$ use terms in $A^{c}$. Unfortunately, choosing a partition based entirely on $\operatorname{cut}(A, B)$ tends to produce unbalanced clusters, i.e., the sizes of $A$ and/or $B$ or their compliments tend to be small. Inspired by the work in $[4,5,14]$, we propose the following normalized variant of the edge cut in (1)

$$
\begin{gathered}
\operatorname{Ncut}(A, B) \equiv \frac{\operatorname{cut}(A, B)}{W(A, Y)+W(X, B)} \\
+\frac{\operatorname{cut}\left(A^{c}, B^{c}\right)}{W\left(A^{c}, Y\right)+W\left(X, B^{c}\right)} .
\end{gathered}
$$

The intuition behind this criterion is that not only we want a partition with small edge cut, but we also want the two subgraphs formed between the matched vertices to be as dense as possible. This latter requirement is partially satisfied by introducing the normalizing denominators in the above equation. ${ }^{2}$ Our bi-clustering problem is now equivalent to the following optimization problem

$$
\min _{\Pi(A, B)} \operatorname{Ncut}(A, B)
$$

i.e., finding partitions of the vertex sets $X$ and $Y$ to minimize the normalized cut of the bipartite graph $G(X, Y, W)$.

\section{APPROXIMATE SOLUTIONS USING SIN- GULAR VECTORS}

Given a bipartite graph $G(X, Y, W)$ and the associated partition $\Pi(A, B)$. Let us reorder the vertices of $X$ and $Y$ so that vertices in $A$ and $B$ are ordered before vertices in $A^{c}$ and $B^{c}$, respectively. The weight matrix $W$ can be written in a block format

$$
W=\left[\begin{array}{ll}
W_{11} & W_{12} \\
W_{21} & W_{22}
\end{array}\right],
$$

i.e., the rows of $W_{11}$ correspond to the vertices in the vertex set $A$ and the columns of $W_{11}$ correspond to those in $B$. Therefore $G\left(A, B, W_{11}\right)$ denotes the weighted bipartite graph corresponding to the vertex sets $A$ and $B$. For any $m$-by- $n$ matrix $H=\left(h_{i j}\right)$, define

$$
\mathrm{s}(H)=\sum_{i=1}^{m} \sum_{j=1}^{n} h_{i j},
$$

i.e., $\mathrm{s}(H)$ is the sum of all the elements of $H$. It is easy to see from the definition of Ncut,

$$
\begin{gathered}
\operatorname{Ncut}(A, B)=\frac{\mathrm{s}\left(W_{12}\right)+\mathrm{s}\left(W_{21}\right)}{2 \mathrm{~s}\left(W_{11}\right)+\mathrm{s}\left(W_{12}\right)+\mathrm{s}\left(W_{21}\right)} \\
+\frac{\mathrm{s}\left(W_{12}\right)+\mathrm{s}\left(W_{21}\right)}{2 \mathrm{~s}\left(W_{22}\right)+\mathrm{s}\left(W_{12}\right)+\mathrm{s}\left(W_{21}\right)} .
\end{gathered}
$$

${ }^{2} \mathrm{~A}$ more natural criterion seems to be

$$
\frac{\operatorname{cut}(A, B)}{W(A, B)}+\frac{\operatorname{cut}\left(A^{c}, B^{c}\right)}{W\left(A^{c}, B^{c}\right)}
$$

However, it can be shown that it will leads to an SVD problem with the same set of left and right singular vectors. 
In order to make connections to SVD problems, we first consider the case when $W$ is symmetric. ${ }^{3}$ It is easy to see that with $W$ symmetric (denoting $\operatorname{Ncut}(A, A)$ by $\operatorname{Ncut}(A))$, we have

$$
\operatorname{Ncut}(A)=\frac{\mathrm{s}\left(W_{12}\right)}{\mathrm{s}\left(W_{11}\right)+\mathrm{s}\left(W_{12}\right)}+\frac{\mathrm{s}\left(W_{12}\right)}{\mathrm{s}\left(W_{22}\right)+\mathrm{s}\left(W_{12}\right)} .
$$

Let $e$ be the vector with all its elements equal to 1 . Let $D$ be the diagonal matrix such that $W e=D e$. Then $(D-W) e=$ 0 . Let $x=\left(x_{i}\right)$ be the vector with

$$
x_{i}=\left\{\begin{aligned}
1, & i \in A \\
-1, & i \in A^{c}
\end{aligned}\right.
$$

It is easy to verify that

$$
\mathrm{s}\left(W_{12}\right)=x^{T}(D-W) x / 4 .
$$

Define

$$
p \equiv \frac{\mathrm{s}\left(W_{11}\right)+\mathrm{s}\left(W_{12}\right)}{\mathrm{s}\left(W_{11}\right)+2 \mathrm{~s}\left(W_{12}\right)+\mathrm{s}\left(W_{22}\right)}=\frac{\mathrm{s}\left(W_{11}\right)+\mathrm{s}\left(W_{12}\right)}{e^{T} D e} .
$$

Then

$$
\begin{gathered}
\mathrm{s}\left(W_{11}\right)+\mathrm{s}\left(W_{12}\right)=p e^{T} D e \\
\mathrm{~s}\left(W_{22}\right)+\mathrm{s}\left(W_{12}\right)=(1-p) e^{T} D e
\end{gathered}
$$

and

$$
\operatorname{Ncut}(A)=\frac{x^{T}(D-W) x}{4 p(1-p) e^{T} D e}
$$

Notice that $(D-W) e=0$, then for any scalar $s$, we have

$$
(s e+x)^{T}(D-W)(s e+x)=x^{T}(D-W) x .
$$

To cast (4) in the form of a Rayleigh quotient, we need to find $s$ such that

$$
(s e+x)^{T} D(s e+x)=4 p(1-p) e^{T} D e .
$$

Since $x^{T} D x=e^{T} D e$, it follows from the above equation that $s=1-2 p$. Now let $y=(1-2 p) e+x$, it is easy to see that $y^{T} D e=((1-2 p) e+x)^{T} D e=0$, and

$$
y_{i}=\left\{\begin{aligned}
2(1-p)>0, & i \in A, \\
-2 p<0, & i \in A^{c} .
\end{aligned}\right.
$$

Thus

$$
\min _{A} \operatorname{Ncut}(A)=\min \left\{\frac{y^{T}(D-W) y}{y^{T} D y} \mid y \in S\right\}
$$

where

$$
S=\left\{y \mid y^{T} D e=0, y_{i} \in\{2(1-p),-2 p\}\right\} .
$$

If we drop the constraints $y_{i} \in\{2(1-p),-2 p\}$ and let the elements of $y$ take arbitrary continuous values, then the optimal $y$ can be approximated by the following relaxed continuous minimization problem,

$$
\min \left\{\frac{y^{T}(D-W) y}{y^{T} D y} \mid y^{T} D e=0\right\} .
$$

Notice that it follows from $W e=D e$ that

$$
D^{-1 / 2} W D^{-1 / 2}\left(D^{1 / 2} e\right)=D^{-1 / 2} e,
$$

${ }^{3} \mathrm{~A}$ different proof for the symmetric case was first derived in [14]. However, our derivation is simpler and more transparent and leads naturally to the SVD problems for the rectangular case. and therefore $D^{1 / 2} e$ is an eigenvector of $D^{-1 / 2} W D^{-1 / 2}$ corresponding to the eigenvalue 1 . It is easy to show that all the eigenvalues of $D^{-1 / 2} W D^{-1 / 2}$ have absolute value at most 1 (See the Appendix). Thus the optimal $y$ in (5) can be computed as $y=D^{1 / 2} \hat{y}$, where $\hat{y}$ is the second largest eigenvector of $D^{-1 / 2} W D^{-1 / 2}$.

Now we return to the rectangular case for the weight matrix $W$, and let $D_{X}$ and $D_{Y}$ be diagonal matrices such that

$$
W e=D_{X} e, \quad W^{T} e=D_{Y} e .
$$

Consider a partition $\Pi(A, B)$, and define

$$
u_{i}=\left\{\begin{array}{rl}
1, & i \in A \\
-1, & i \in A^{c}
\end{array} \quad, \quad v_{i}=\left\{\begin{aligned}
1, & i \in B \\
-1, & i \in B^{c}
\end{aligned}\right.\right.
$$

Let $W$ have the block form as in (2), and consider the augmented symmetric matrix ${ }^{4}$

$$
\hat{W}=\left[\begin{array}{cc}
0 & W \\
W^{T} & 0
\end{array}\right]=\left[\begin{array}{cc|cc}
0 & 0 & W_{11} & W_{12} \\
0 & 0 & W_{21} & W_{22} \\
\hline W_{11}^{T} & W_{21}^{T} & 0 & 0 \\
W_{12}^{T} & W_{22}^{T} & 0 & 0
\end{array}\right] .
$$

If we interchange the second and third block rows and columns of the above matrix, we obtain

$$
\left[\begin{array}{cc|cc}
0 & W_{11} & 0 & W_{12} \\
W_{11}^{T} & 0 & W_{21}^{T} & 0 \\
\hline 0 & W_{21} & 0 & W_{22} \\
W_{12}^{T} & 0 & W_{22}^{T} & 0
\end{array}\right] \equiv\left[\begin{array}{cc}
\hat{W}_{11} & \hat{W}_{12} \\
\hat{W}_{12}^{T} & \hat{W}_{22}
\end{array}\right]
$$

and the normalized cut can be written as

$$
\operatorname{Ncut}(A, B)=\frac{\mathrm{s}\left(\hat{W}_{12}\right)}{\mathrm{s}\left(\hat{W}_{11}\right)+\mathrm{s}\left(\hat{W}_{12}\right)}+\frac{\mathrm{s}\left(\hat{W}_{12}\right)}{\mathrm{s}\left(\hat{W}_{22}\right)+\mathrm{s}\left(\hat{W}_{12}\right)},
$$

a form that resembles the symmetric case (3). Define

$$
q=\frac{2 \mathrm{~s}\left(W_{11}\right)+\mathrm{s}\left(W_{12}\right)+\mathrm{s}\left(W_{21}\right)}{e^{T} D_{X} e+e^{T} D_{Y} e} .
$$

Then we have

$$
\begin{aligned}
\operatorname{Ncut}(A, B) & =\frac{-2 x^{T} W y+x^{T} D_{X} x+y^{T} D_{Y} y}{x^{T} D_{X} x+y^{T} D_{Y} y} \\
& =1-\frac{2 x^{T} W y}{x^{T} D_{X} x+y^{T} D_{Y} y},
\end{aligned}
$$

where $x=(1-2 p) e+u, y=(1-2 p) e+v$. It is also easy to see that

$$
x^{T} D_{X} e+y^{T} D_{Y} e=0, \quad x_{i}, y_{i} \in\{2(1-q),-2 q\} .
$$

Therefore,

$$
\begin{gathered}
\min _{\Pi(A, B)} \operatorname{Ncut}(A, B) \\
=1-\max _{x \neq 0, y \neq 0}\left\{\frac{2 x^{T} W y}{x^{T} D_{X} x+y^{T} D_{Y} y} \mid x, y \text { satisfy (7) }\right\} .
\end{gathered}
$$

${ }^{4}$ In [11], the Laplacian of $\hat{W}$ is used for partitioning a rectangular matrix in the context of designing load-balanced matrix-vector multiplication algorithms for parallel computation. However, the eigenvalue problem of the Laplacian of $\hat{W}$ does not lead to a simpler singular value problem. 
Ignoring the discrete constraints on the elements of $x$ and $y$, we have the following continuous maximization problem,

$$
\max _{x \neq 0, y \neq 0}\left\{\frac{2 x^{T} W y}{x^{T} D_{X} x+y^{T} D_{Y} y} \mid x^{T} D_{X} e+y^{T} D_{Y} e=0\right\} .
$$

Without the constraints $x^{T} D_{X} e+y^{T} D_{Y} e=0$, the above problem is equivalent to computing the largest singular triplet of $D_{X}^{-1 / 2} W D_{Y}^{-1 / 2}$ (see the Appendix). From (6), we have

$$
\begin{gathered}
D_{X}^{-1 / 2} W D_{Y}^{-1 / 2}\left(D_{Y}^{1 / 2} e\right)=D_{X}^{1 / 2} e \\
\left(D_{X}^{-1 / 2} W D_{Y}^{-1 / 2}\right)^{T}\left(D_{X}^{1 / 2} e\right)=D_{Y}^{1 / 2} e,
\end{gathered}
$$

and similarly to the symmetric case, it is easy to show that all the singular values of $D_{X}^{-1 / 2} W D_{Y}^{-1 / 2}$ are at most 1 . Therefore, an optimal pair $\{x, y\}$ for (8) can be computed as $x=D_{X}^{-1 / 2} \hat{x}$ and $y=D_{Y}^{-1 / 2} \hat{y}$, where $\hat{x}$ and $\hat{y}$ are the $s e c$ ond largest left and right singular vectors of $D_{X}^{-1 / 2} W D_{Y}^{-1 / 2}$, respectively (see the Appendix). With the above discussion, we can now summerize our basic approach for bipartite graph clustering incorporating a recursive procedure.

Algorithm. Spectral Recursive Embedding (SRE) Given a weighted bipartite graph $G=(X, Y, E)$ with its edge weight matrix $W$ :

1. Compute $D_{X}$ and $D_{Y}$ and form the scaled weight matrix $\hat{W}=D_{X}^{-1 / 2} W D_{Y}^{-1 / 2}$.

2. Compute the second largest left and right singular vectors of $\hat{W}, \hat{x}$ and $\hat{y}$.

3. Find cut points $c_{x}$ and $c_{y}$ for $x=D_{X}^{-1 / 2} \hat{x}$ and $y=D_{Y}^{-1 / 2} \hat{y}$, respectively.

4. Form partitions $A=\left\{i \quad \mid x_{i} \geq c_{x}\right\}$ and $A^{c}=\left\{i \mid x_{i}<c_{x}\right\}$ for vertex set $X$, and $B=\left\{j \mid y_{j} \geq c_{y}\right\}$ and $B^{c}=\left\{j \mid y_{j}<c_{y}\right\}$ for vertex set $Y$.

5. Recursively partition the sub-graphs $G(A, B)$ and $G\left(A^{c}, B^{c}\right)$ if necessary.

Two basic strategies can be used for selecting the cut points $c_{x}$ and $c_{y}$. The simplest strategy is to set $c_{x}=0$ and $c_{y}=0$. Another more computing-intensive approach is to base the selection on Ncut: Check $N$ equally spaced splitting points of $x$ and $y$, respectively, find the cut points $c_{x}$ and $c_{y}$ with the smallest Ncut [14].

Computational complexity. The major computational cost of SRE is Step 2 for computing the left and right singular vectors which can be obtained either by power method or more robustly by Lanczos bidiagonalization process [8, Chapter 9]. Lanczos method is an iterative process for computing partial SVDs in which each iterative step involves the computation of two matrix-vector multiplications $\hat{W} u$ and $\hat{W}^{T} v$ for some vectors $u$ and $v$. The computational cost of these is roughly proportional to $\operatorname{nnz}(\hat{W})$, the number of nonzero elements of $\hat{W}$. The total computational cost of SRE is $O\left(c_{\text {sre }} k_{\text {svd }} \mathrm{nnz}(\hat{W})\right)$, where $c_{\text {sre }}$ the the level of recursion and $k_{\mathrm{svd}}$ is the number of Lanczos iteration steps.
In general, $k_{\mathrm{svd}}$ depends on the singular value gaps of $\hat{W}$. Also notice that $\operatorname{nnz}(\hat{W})=n_{w} n$, where $n_{w}$ is the average number of terms per document and $n$ is the total number of document. Therefore, the total cost of SRE is in general linear in the number of documents to be clustered.

\section{CONNECTIONS TO CORRESPONDENCE ANALYSIS}

In its basic form correspondence analysis is applied to an $m$-by- $n$ two-way table of counts $W[2,10,16]$. Let $w=\mathrm{s}(W)$, the sum of all the elements of $W, D_{X}$ and $D_{Y}$ be diagonal matrices defined in section 3. Correspondence analysis seeks to compute the largest singular triplets of the matrix $Z=\left(z_{i j}\right) \in \mathcal{R}^{m \times n}$ with

$$
z_{i j}=\frac{w_{i j} / w-\left(D_{X}(i, i) / w\right)\left(D_{Y}(j, j) / w\right)}{\sqrt{\left(D_{X}(i, i) / w\right)\left(D_{Y}(j, j) / w\right)}} .
$$

The matrix $Z$ can be considered as the correlation matrix of two group indicator matrices for the original $W[16]$. We now show that the SVD of $Z$ is closely related to the SVD of $\hat{W} \equiv D_{X}^{-1 / 2} W D_{Y}^{-1 / 2}$. In fact, in section 3 , we showed that $D_{X}^{1 / 2} e$ and $D_{Y}^{1 / 2} e$ are the left and right singular vectors of $\hat{W}$ corresponding to the singular value one, and it is also easy to show that all the singular values of $\hat{W}$ are at most 1. Therefore, the rest of the singular values and singular vectors of $\hat{W}$ can be found by computing the SVD of the following rank-one modification of $\hat{W}$

$$
D_{X}^{-1 / 2} W D_{Y}^{-1 / 2}-\frac{D_{X}^{1 / 2} e e^{T} D_{Y}^{1 / 2}}{\left\|D_{X}^{1 / 2} e\right\|_{2}\left\|D_{Y}^{1 / 2}\right\|_{2}}
$$

which has $(i, j)$ element

$$
\frac{w_{i j}}{\sqrt{D_{X}(i, i) D_{Y}(j, j)}}-\frac{\sqrt{D_{X}(i, i) D_{Y}(j, j)}}{w}=w^{2} z_{i j},
$$

and is a constant multiple of the $(i, j)$ element of $Z$. Therefore, normalized-cut based cluster analysis and correspondence analysis arrive at the same SVD problems even though they start with completely different principles. It is worthwhile to explore more deeply the interplay between these two different points of views and approaches, for example, using the statistical analysis of correspondence analysis to provide better strategy for selecting cut points and estimating the number of clusters.

\section{PARTITIONS WITH OVERLAPS}

So far in our discussion, we have only looked at hard clustering, i.e., a data object belongs to one and only one cluster. In many situations, especially when there are much overlap among the clusters, it is more advantageous to allow data objects to belong to different clusters. For example, in document clustering, certain groups of words can be shared by two clusters. Is it possible to model this overlap using our bipartite graph model and also find efficient approximate solutions? The answer seems to be yes, but our results at this point are rather preliminary and we will only illustrate the possibilities. Our basic idea is that when computing $\operatorname{Ncut}(A, B)$, we should disregard the contributions of the set of vertices that is in the overlap. More specifically, let $X=A \cup O_{X} \cup \bar{A}$ and $Y=B \cup O_{Y} \cup \bar{B}$, where $O_{X}$ denotes the 

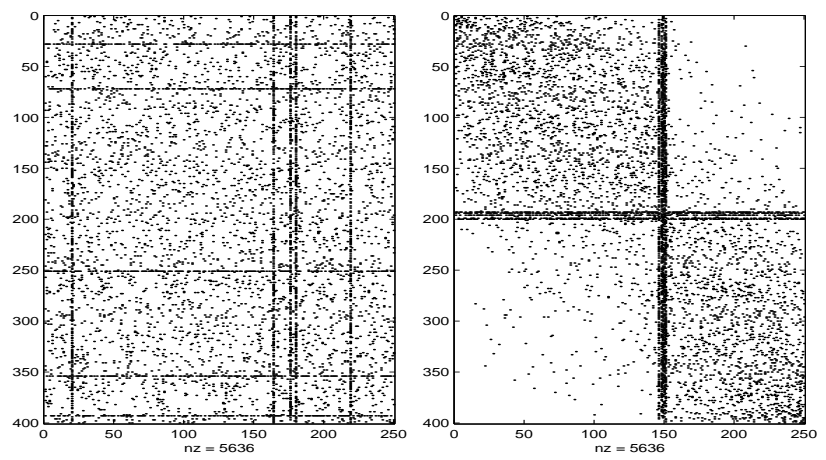

Figure 1: Sparsity patterns of a test matrix before clustering (left) and after clustering (right)

overlap between the vertex subsets $A \cup O_{X}$ and $\bar{A} \cup O_{X}$, and $O_{Y}$ the overlap between $B \cup O_{Y}$ and $\bar{B} \cup O_{Y}$, we compute

$$
\begin{gathered}
\operatorname{Ncut}(A, B, \bar{A}, \bar{B})=\frac{\operatorname{cut}(A, B)}{W(A, Y)+W(X, B)} \\
+\frac{\operatorname{cut}(\bar{A}, \bar{B})}{W(\bar{A}, Y)+W(X, \bar{B})} .
\end{gathered}
$$

However, we can make $\operatorname{Ncut}(A, B, \bar{A}, \bar{B})$ smaller simply by putting more vertices in the overlap. Therefore, we need to balance these two competing quantities: the size of the overlap and the modified normalized cut $\operatorname{Ncut}(A, B, \bar{A}, \bar{B})$ by minimizing

$$
\operatorname{Ncut}(A, B, \bar{A}, \bar{B})+\alpha\left(\left|O_{X}\right|+\left|O_{Y}\right|\right),
$$

where $\alpha$ is a regularization parameter. How to find an efficient method for computing the (approximate) optimal solution to the above minimization problem still needs to be investigated. We close this section by presenting an illustrative example showing that in some situations, the singular vectors already automatically separating the overlap sets while giving the coordinates for carrying out clustering.

EXAMPLE 1 . We construct a sparse $m$-by- $n$ rectangular matrix

$$
W=\left[\begin{array}{ll}
W_{11} & W_{12} \\
W_{21} & W_{22}
\end{array}\right] .
$$

so that $W_{11}$ and $W_{22}$ are relatively denser than $W_{12}$ and $W_{21}$. We also add some dense rows and columns to the matrix $W$ to represent row and column overlaps. The left panel of Figure 1 shows the sparsity pattern of $\bar{W}$, a matrix obtained by randomly permuting the rows and columns of $W$. We then compute the second largest left and right singular vectors of $D_{X}^{-1 / 2} \bar{W} D_{Y}^{-1 / 2}$, say $x$ and $y$, then sort the rows and columns of $\bar{W}$ according to the values of the entries in $D_{X}^{-1 / 2} x$ and $D_{\underline{Y}}^{-1 / 2} y$, respectively. The sparsity pattern of this permuted $\bar{W}$ is shown on the right panel of Figure 1. As can be seen that the singular vectors not only do the job of clustering but at the same time also concentrate the dense rows and columns at the boundary of the two clusters.

\section{EXPERIMENTS}

In this section we present our experimental results on clustering a dataset of newsgroup articles submitted to 20 news- groups. ${ }^{5}$ This dataset contains about 20,000 articles (email messages) evenly divided among the 20 newsgroups. We list the names of the newsgroups together with the associated group labels (the labels will be used in the sequel to identify the newsgroups).

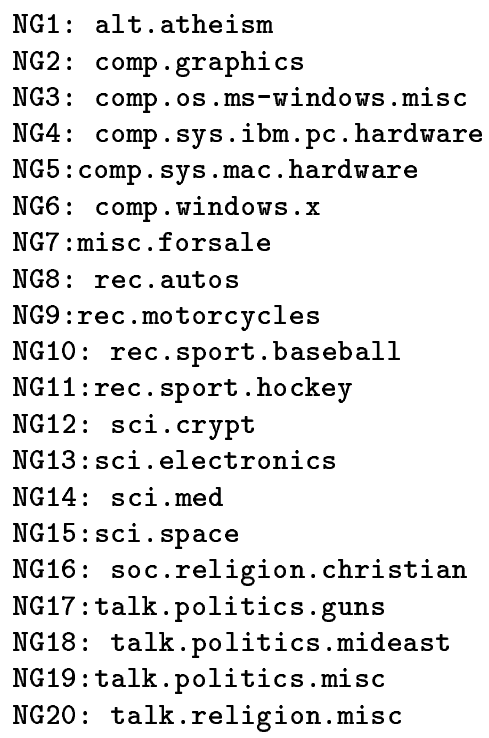

We used the bow toolkit to construct the term-document matrix for this dataset, specifically we use the tokenization option so that the UseNet headers are stripped, and we also applied stemming [13]. Some of the newsgroups have large overlaps, for example, the five newsgroups comp.* about computers. In fact several articles are posted to multiple newsgroups. Before we apply clustering algorithms to the dataset, several preprocessing steps need to be considered. Two standard steps are weighting and feature selection. For weighting, we considered a variant of tf.idf weighting scheme, tf $\log _{2}(n / \mathrm{df})$, where tf is the term frequency and $\mathrm{df}$ is the document frequency and several other variations listed in [1]. For feature selection, we looked at three approaches 1) deleting terms that occur less than certain number of times in the dataset; 2) deleting terms that occur in less than certain number of documents in the dataset; 3 ) selecting terms according to mutual information of terms and documents defined as

$$
I(y)=\sum_{x} p(x, y) \log (p(x, y) /(p(x) p(y)),
$$

where $y$ represents a term and $x$ a document [15]. In general we found out that the traditional tf.idf based weighting schemes do not improve performance for SRE. One possible explanation comes from the connection with correspondence analysis, the raw frequencies are samples of co-occurrence probabilities, and the pre- and post-multiplication by $D_{X}^{-1 / 2}$ and $D_{Y}^{-1 / 2}$ in $D_{X}^{-1 / 2}(D-W) D_{Y}^{-1 / 2}$ automatically taking into account of weighting. We did, however, found out that trimming the raw frequencies can sometimes improve performance for SRE, especially for the anomalous cases where some words can occur in certain documents an unusual number of times, skewing the clustering process.

\footnotetext{
${ }^{5}$ The newsgroup dataset together with the bow toolkit for processing it can be downloaded from http://www.cs.cmu.edu/afs/cs/project/theo-11/www/ naive-bayes.html.
} 
Table 1: Comparison of spectral embedding (SRE), PDDP, and K-means (NG1/NG2)

\begin{tabular}{llrr} 
Mixture & SRE & PDDP & K-means \\
\hline \hline $50 / 50$ & $92.12 \pm 3.52 \%$ & $91.90 \pm 3.19 \%(53,10,37)$ & $76.93 \pm 14.42 \%(82,2,10)$ \\
\hline $50 / 100$ & $90.57 \pm 3.11 \%$ & $86.11 \pm 3.94 \%(86,5,9)$ & $76.74 \pm 14.01 \%(80,2,18)$ \\
\hline $50 / 150$ & $88.04 \pm 3.90 \%$ & $78.60 \pm 5.03 \%(98,0,2)$ & $68.80 \pm 13.55 \%(88,0,12)$ \\
\hline $50 / 200$ & $82.77 \pm 5.24 \%$ & $70.43 \pm 6.04 \%(97,0,3)$ & $69.22 \pm 12.34 \%(83,1,16)$ \\
\hline
\end{tabular}

Table 2: Comparison of spectral embedding (SRE), PDDP, and K-means (NG10/NG11)
Mixture
\begin{tabular}{llrrr} 
SRE & PDDP & K-means \\
\hline \hline $50 / 50$ & $74.56 \pm 8.93 \%$ & $73.40 \pm 10.07 \%(56,6,38)$ & $61.61 \pm 8.77 \%(86,0,14)$ \\
\hline $50 / 100$ & $67.13 \pm 7.17 \%$ & $67.10 \pm 10.20 \%(52,1,47)$ & $64.40 \pm 9.37 \%(59,1,40)$ \\
\hline $50 / 150$ & $58.30 \pm 5.99 \%$ & $58.72 \pm 7.48 \%(52,1,47)$ & $62.53 \pm 8.20 \%(36,1,63)$ \\
\hline $50 / 200$ & $57.55 \pm 5.69 \%$ & $56.63 \pm 4.84 \%(58,1,41)$ & $60.82 \pm 7.54 \%(39,2,59)$ \\
\hline
\end{tabular}

For the purpose of comparison, we consider two other clustering methods: 1) K-means method [9]; 2) Principal direction divisive partion (PDDP) method [3]. K-means method is a widely used cluster analysis tool. The variant we used employs the Euclidean distance when comparing the dissimilarity between two documents. When applying K-means, we normalize the length of each document so that it has Euclidean length one. In essence, we use the cosine of the angle between two document vectors when measuring their similarity. We have also tried K-means without document length normalization, the results are far worse and therefore we will not report the corresponding results. Since K-means method is an iterative method, we need to specify a stopping criterion. For the variant we used, we compare the centroids between two consecutive iterations, and stop when the difference is smaller than a pre-defined tolerance.

PDDP is another clustering method that utilizes singular vectors. It is based on the idea of principal component analysis and has been shown to outperform several standard clustering methods such as hierarchical agglomerative algorithm [3]. First each document is considered as a multivariate data point. The set of document is normalized to have unit Euclidean length and then centered, i,e., let $W$ be the term-document matrix, and $w$ be the average of the columns of $W$. Compute the largest singular value triplet $\{u, \sigma, v\}$ of $W-w e^{T}$. Then split the set of documents based on their values of the $v=\left(v_{i}\right)$ vector: one simple scheme is to let those with positive $v_{i}$ go into one cluster and those with nonnegative $v_{i}$ inot another cluster. Then the whole process is repeated on the term-document matrices of the two clusters, respectively. Although both our clustering method SRE and PDDP make use of the singular vectors of some versions of the term-document matrices, they are derived from fundamentally different principles. PDDP is a featurebased clustering method, projecting all the data points to the one-dimensional subspace spanned by the first principal axis; SRE is a similarity-based clustering method, two cooccurring variables (terms and documents in the context of document clustering) are simultaneously clustered. Unlike SRE, PDDP does not have a well-defined objective function for minimization. It only partitions the columns of the termdocument matrices while SRE partitions both of its rows and columns. This will have significant impact on the computational costs. PDDP, however, has an advantage that it can be applied to dataset with both positive and negative values while SRE can only be applied to datasets with nonnegative data values.

EXAMPLE 2. In this example, we examine binary clustering with uneven clusters. We consider three pairs of newsgroups: newsgroups 1 and 2 are well-separated, 10 and 11 are less well-separated and 18 and 19 have a lot of overlap. We used document frequency as the feature selection criterion and delete words that occur in less than 5 documents in each datasets we used. For both K-means and PDDP we apply tf.idf weighting together with document length normalization so that each document vector will have Euclidean norm one. For SRE we trim the raw frequency so that the maximum is 10 . For each newsgroup pair, we select four types of mixture of articles from each newsgroup: $x / y$ indicates that $x$ articles are from the first group and $y$ articles are from the second group. The results are listed in Table 1 for groups 1 and 2, Table 2 for groups 10 and 11 and Table 3 for groups 18 and 19. We list the means and standard deviations for 100 random samples. For PDDP and K-means we also include a triplet of numbers which indicates how many of the 100 samples SRE performs better (the first number), the same (the second number) and worse (the third number) than the corresponding methods (PDDP or K-means). We should emphasize that K-means method can only find local minimum, and the results depend on initial values and stopping criteria. This is also reflected by the large standard deviations associated with K-means method. From the three tests we can conclude that both SRE and PDDP outperform K-means method. The performance of SRE and PDDP are similar in balanced mixtures, but SRE is superior to PDDP in skewed mixtures.

EXAMPLE 3. In this example, we consider an easy multicluster case, we examine five newsgroups $2,9,10,15,18$ which was also considered in [15]. We sample 100 articles from each newsgroups, we use mutual information for feature selection. We use minimum normalized cut as cut point for each level of the recursion. For one sample, Table 4 gives the confusion matrix. The accuracy for this sample is $88.2 \%$. We also tested two other samples with accuracy $85.4 \%$ and $81.2 \%$ which compare favorably with those obtained for three samples with accuracy $59 \%, 58 \%$ and $53 \%$ reported in [15]. In the following we also listed the top few words for each clusters computed by mutual information. 
Table 3: Comparison of spectral embedding (SRE), PDDP, and K-means (NG18/NG19)

\begin{tabular}{llrr} 
Mixture & SRE & PDDP & K-means \\
\hline \hline $50 / 50$ & $73.66 \pm 10.53 \%$ & $69.52 \pm 12.83 \%(65,12,32)$ & $62.25 \pm 9.94 \%(82,1,17)$ \\
\hline $50 / 100$ & $67.23 \pm 7.84 \%$ & $67.84 \pm 7.30 \%(46,5,49)$ & $60.91 \pm 7.92 \%(65,13,32)$ \\
\hline $50 / 150$ & $65.83 \pm 12.79 \%$ & $60.37 \pm 9.85 \%(53,3,44)$ & $63.32 \pm 8.26 \%(58,3,39)$ \\
\hline $50 / 200$ & $61.23 \pm 9.88 \%$ & $60.76 \pm 5.55 \%(40,1,59)$ & $64.50 \pm 7.58 \%(34,0,66)$ \\
\hline
\end{tabular}

Table 4: Confusion matrix for newsgroups $\{2,9,10,15,18\}$

\begin{tabular}{|l||c|c|c|c|c|}
\hline \hline & mideast & graphics & space & baseball & motorcycles \\
\hline \hline cluster 1 & 87 & 0 & 0 & 2 & 0 \\
\hline cluster 2 & 7 & 90 & 7 & 6 & 7 \\
\hline cluster 3 & 3 & 9 & 84 & 1 & 1 \\
\hline cluster 4 & 0 & 0 & 1 & 88 & 0 \\
\hline cluster 5 & 3 & 1 & 8 & 3 & 92 \\
\hline \hline
\end{tabular}

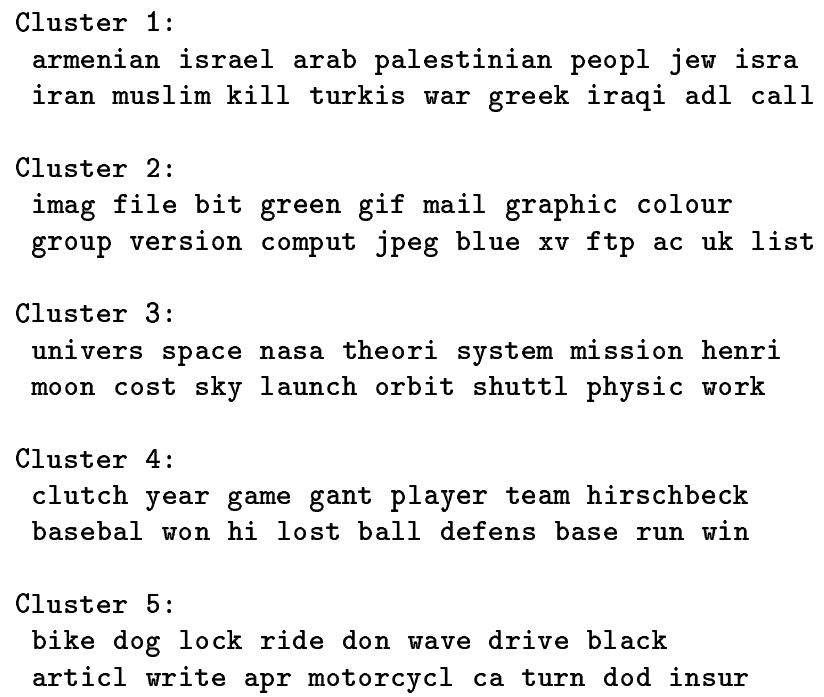

\section{CONCLUSIONS AND FUTURE WORK}

In this paper, we formulate a class of clustering problems as bipartite graph partitioning problems, and we show that efficient optimal solutions can be found by computing the partial singular value decomposition of some scaled edge weight matrices. However, we have also shown that there still remain many challenging problems. One area that needs further investigation is the selection of cut points and number of clusters using multiple left and right singular vectors, and the possibility of adding local refinements to improve clustering quality. ${ }^{6}$ Another area is to find efficient algorithms for handling overlapping clusters. Finally, the treatment of missing data under our bipartite graph model especially when we apply our spectral clustering methods to the problem of data analysis of recommender systems also deserves further investigation.

\section{ACKNOWLEDGMENTS}

\footnotetext{
${ }^{6}$ It will be difficult to use local refinement for PDDP because it does not have a global objective function for minimization.
}

The work of Hongyuan Zha and Xiaofeng He was supported in part by NSF grant CCR-9901986. The work of Xiaofeng He, Chris Ding and Horst Simon was supported in part by Department of Energy through an LBL LDRD fund.

\section{REFERENCES}

[1] R. Belew. Finding Out About: A Cognitive Perspective on Search Engine Technology and the $W W W$. Cambridge University Press, 2000.

[2] J.-P. Benzecri. Correspondence analysis handbook. Marcel Dekker, 1992.

[3] D. L. Boley. Principal Direction Divisive Partitioning. Data Mining and Knowledge Discovery, 2(4):325-344, 1998.

[4] F.R.K. Chung. Spectral Graph Theory. American Mathematical Society, 1997.

[5] R. V. Driessche and D. Roose. An improved spectral bisection algorithm and its application to dynamic load balancing. Parallel Computing, 21:29-48, 1995.

[6] B. Everitt. Cluster Analysis. Edward Arnold, 1993.

[7] M. Fiedler. Algebraic connectivity of graphs. Czechoslovak Math J., 23:298-305, 1973.

[8] G. H. Golub and C. F. Van Loan, Matrix computations, 3nd ed., Johns Hopkins University Press, Baltimore, Maryland, 1996.

[9] A. D. Gordon. Classification. Second Edition, Chapman and Hall, 2000.

[10] M.J. Greenacre. Correspondence analysis in practice. Academic Press, 1993.

[11] B. Hendrickson and T.G. Kolda. Partitioning sparse rectangular and structurally nonsymmetric matrices for parallel computation. SIAM J. Scientific Computing, 21:2048-2072, 2000.

[12] T. Hofmann. Probabilistic Latent Semantic Indexing. Proceedings of the 22nd International Conference on Research and Development in Information Retrieval (SIGIR'99), 1999.

[13] A. McCallum. Bow: A toolkit for statistical language modeling, text retrieval, classification and clustering. http://www.cs.cmu.edu/ mccallum/bow, 1996. 
[14] J. Shi and J. Malik. Normalized cuts and image segmentation. Proc. of the IEEE Conf. on Computer Vision and Pattern Recognition, June 1997.

[15] N. Slonim and N. Tishby Document clustering using word clusters via the information bottleneck method. Proceedings of SIGIR-2000, 2000.

[16] W.N. Venables and B.D. Ripley. Modern Applied Statistics with S-plus. Springer-verlag, 1999.

\section{APPENDIX}

\section{A. SOME PROOFS}

In this appendix we prove three results: 1) All the eigenvalues of $D^{-1 / 2} W D^{-1 / 2}$ has absolute value at most 1 . Equivalently, we need to prove that the eigenvalues of the generalized eigenvalue problem $W x=\lambda D x$ has absolute value at most 1 . In fact let $x=\left(x_{i}\right)_{i=1}^{n}$ and let $i$ be such that $\left|x_{i}\right|=\max \left|x_{j}\right|$, then it follows from

$$
\lambda d_{i} x_{i}=\sum_{j=1}^{n} w_{i j} x_{j}
$$

that

$$
|\lambda| \leq \sum_{j=1}^{n} w_{i j} / d_{i}=1 .
$$

2) We prove that

$$
\sigma_{\max }\left(D_{X}^{-1 / 2} W D_{Y}^{-1 / 2}\right)=\max _{x \neq 0, y \neq 0} \frac{2 x^{T} W y}{x^{T} D_{X} x+y^{T} D_{Y} y} .
$$

Let $\hat{x}=D_{X}^{1 / 2} x$ and $\hat{y}=D_{Y}^{1 / 2} y$, then

$$
\frac{2 x^{T} W y}{x^{T} D_{X} x+y^{T} D_{Y} y}=\frac{2 \hat{x}^{T} D_{X}^{-1 / 2} W D_{Y}^{-1 / 2} \hat{y}}{\hat{x}^{T} \hat{x}+\hat{y}^{T} \hat{y}} .
$$

Let $D_{X}^{-1 / 2} W D_{Y}^{-1 / 2}=U \Sigma V^{T}$ be its SVD with

$$
U=\left[u_{1}, \ldots, u_{m}\right], \quad V=\left[v_{1}, \ldots, v_{n}\right]
$$

and

$$
\Sigma=\operatorname{diag}\left(\sigma_{1}, \ldots, \sigma_{\min \{m, n\}}\right), \quad \sigma_{1}=\sigma_{\max }\left(D_{X}^{-1 / 2} W D_{Y}^{-1 / 2}\right) .
$$

Then we can expand $\hat{x}$ and $\hat{y}$ as

$$
\hat{x}=\sum_{i} \hat{x}_{i} u_{i}, \quad \hat{y}=\sum_{i} \hat{y}_{i} v_{i}
$$

and (9) becomes

$$
\frac{2 \sum_{i} \sigma_{i} \hat{x}_{i} \hat{y}_{i}}{\sum_{i} \hat{x}_{i}^{2}+\sum_{i} \hat{y}_{i}^{2}} \leq \frac{2 \sigma_{1} \sqrt{\sum_{i} \hat{x}_{i}^{2}} \sqrt{\sum_{i} \hat{y}_{i}^{2}}}{\sum_{i} \hat{x}_{i}^{2}+\sum_{i} \hat{y}_{i}^{2}} \leq \sigma_{1} .
$$

Taking $\hat{x}_{1}=1$ and $\hat{y}_{1}=1$ achieves the maximum.

3) Now we consider the constraint

$$
x^{T} D_{X} e+y^{T} D_{Y} e=0
$$

which is equivalent to $\hat{x}_{1}+\hat{y}_{1}=0$ using the expansions in (10). We can always scale the vectors $\hat{x}$ and $\hat{y}$ without changing the maximum so that $\hat{x}_{1} \geq 0$ and $\hat{y}_{1} \geq 0$. Hence $\hat{x}_{1}+\hat{y}_{1}=0$ implies that $\hat{x}_{1}=\hat{y}_{1}=0$. It is then easy to see that

$$
\sigma_{2}=\max \left\{\frac{2 x^{T} W y}{x^{T} D_{X} x+y^{T} D_{Y} y} \mid x^{T} D_{X} e+y^{T} D_{Y} e=0\right\},
$$

and the maximum is achieved by the second largest left and right singular vectors of $D_{X}^{-1 / 2} W D_{Y}^{-1 / 2}$. 